\title{
Juvenile Geographic Tongue with Atopic Dermatitis-A Case Report
}

\author{
Valen Dela D'souza1*, Prasanna Kumar Rao², Raghavendra Kini ${ }^{3}$, Gowri P Bhandarkar ${ }^{4}$, Roopashri Rajesh Kashyap ${ }^{4}$ \\ and Preethi A Poonja ${ }^{5}$
}

${ }^{1}$ Postgraduate Student, Department of Oral Medicine and Radiology, A J Institute of Dental Sciences, Kuntikana, Mangaluru, Karnataka, India ${ }^{2}$ Professor, Department of Oral Medicine and Radiology, A J Institute of Dental Sciences, Kuntikana, Mangaluru, Karnataka, India ${ }^{3}$ Professor and Head, Department of Oral Medicine and Radiology, A J Institute of Dental Sciences, Kuntikana, Mangaluru, Karnataka, India ${ }^{4}$ Reader, Department of Oral Medicine and Radiology, A J Institute of Dental Sciences, Kuntikana, Mangaluru, Karnataka, India ${ }^{5}$ Assistant Professor, Department of Oral Medicine and Radiology, A J Institute of Dental Sciences, Kuntikana, Mangaluru, Karnataka, India Received: 畊 February 23,2018; Published: 眥 February 28, 2018

*Corresponding author: ValenDelaD’ souza, Department of Oral Medicine \& Radiology, AJ Institute of Dental Sciences Kuntikana, Kuntikana, India

\section{Abstract}

Geographic tongue is a benign recurrent condition characterized by loss of epithelium especially filiform papillae commonly seen in adults. The clinical presentation may vary from asymptomatic to painful, burning ulceration. This paper reports a case of asymptomatic Geographic tongue in a male child with atopic dermatitis.

Abbreviations: GT: Geographic Tongue; HLA: Human Leukocyte Antigens

\section{Introduction}

Geographic tongue is a chronic, inflammatory, and immunemediated benign oral lesion also referred to as benign migratory glossitis, stomatitis areata migrans, erythema migrans, annulus migrans and geographic stomatitis. It was first described as wandering rash of the tongue by Reiter in 1831. The lesion is characterized by serpiginous white lines surrounding areas of smooth, depapillated mucosa [1] The lesion persists for a period of time of several days to weeks and disappears only to reappear at a different location with a different pattern [2]. Here we report a case of Geographic tongue (GT) in a six-year-old patient.

\section{Case Report}

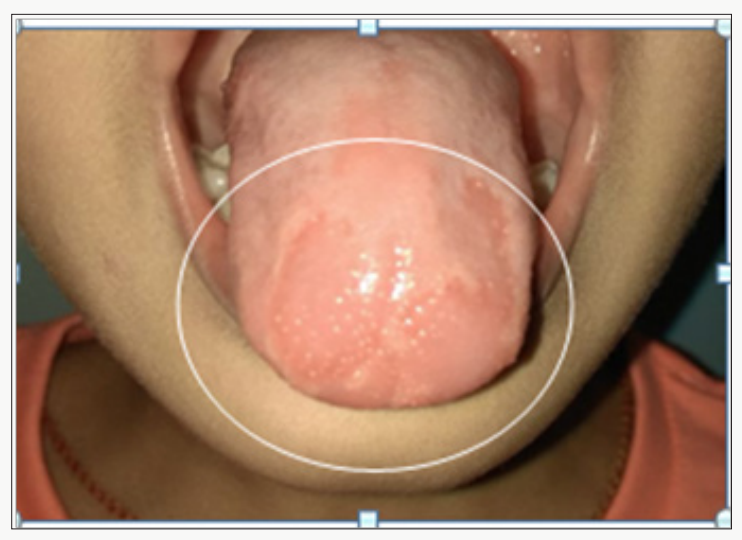

Figure 1: Geographic tongue with oblate pattern.
A six-year-old boy reported to our dental OPD with the chief complaint of carious teeth. Oral examination revealed dentinal caries in relation to the right and left deciduous second molar. Lesions characteristic of GT were found on the anterior two thirds of the dorsal surface as well as the lateral surface of the tongue. Oblate pattern was observed (Figure 1). The patient and his parents were unaware of the condition and no contributing history was elicited. However skin lesions were observed on the limbs, which were diagnosed as atopic dermatitis by a physician (Figure 2). Since the patient was asymptomatic and since GT is a self-limiting condition, reassurance, restoration of carious teeth and oral hygiene maintenance was advised.

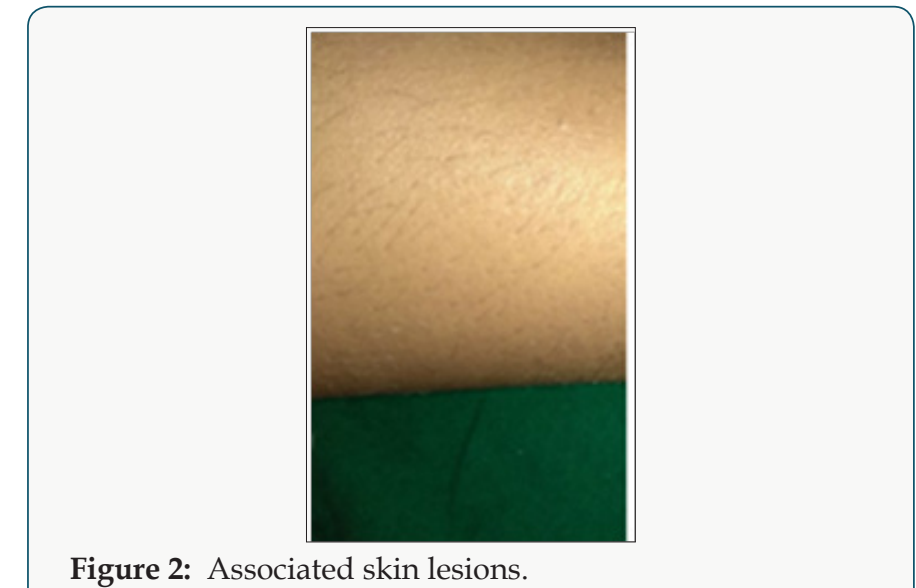




\section{Discussion}

The prevalence of GT in the Indian population is $16.4 \%$ [3]. In children the prevalence is $0.89 \%$ [4]. The highest incidence of about $39.4 \%$ occurs in the age group of $20-29$ years. Some studies show that it is more frequently reported in adults than in children. Females are more commonly affected than males [3]. The reason for low prevalence in the pediatric age group is because it is ignored by the parents due to its asymptomatic nature [1]. This case report is unique since it presents GT in patient who is a male child. It has been classified by some investigators as a congenital anomaly while others have said it to be a hereditary disorder [4]. The occurrence of a similar condition in a study conducted on monozygotic twins might indicate the possible role of genetic factors and this warrants further investigation [5]. Few authors consider it to be a chronic inflammatory condition.The etiological factors include systemic diseases such as atopy, allergy [6], stress, anaemia, psoriasis, gastrointestinal disturbances and hormonal variations. It has been associated with syndromes like Reiter's syndrome, Down syndrome, Aarskog syndrome, Fetal hydantoin syndrome and Robinow's syndrome [4]. In a recent study it has been concluded that immunologic and psycho logic parameters may constitute as risk factor [1] .Deficiency of vitamin B6, B12, folic acid, iron and zinc have been proposed to be contributing factors as well. Drug induced geographic tongue may develop as a result of hormonal fluctuation. A protective influence of smoking has been observed since smokers had less prevalence of geographic tongue than non-smokers [4]. It has been proposed that benign migratory glossitis is an isolated oral manifestation of psoriasis rather than a mere association. The relationship of human leukocyte antigens (HLA) with psoriasis has been extensively investigated. Associations of HLA- Cw6 presents a particularly strong association irrespective of the racial or ethnic group suggesting that Cw6 itself or closely linked gene in strong linkage disequilibrium, is the major HLA-linked susceptibility gene for Psoriasis. Association between GT and fissured tongue is seen in $34.5 \%$ cases [6]. The presence of deep fissures infected with Candida organisms could be responsible for symptom. However, this has to be further investigated [4]. However in our reported case neither Psoriasis nor fissured tongue was seen.

Recently in 2015, GT has been explained in terms of media dynamics based on physics. There are different evolutionary phases of GT: a healed -resting state, a highly inflamed -excited state, and a healing -recovering state. There exist different patterns of GToblate pattern, wavy pattern and a ring shaped concentric pattern, oblate pattern being observed in the case reported here. In circular pattern, shape of lesions will remain same as they expand on tongue unless there is some obstacle or inhomogenity in the epithelium. If circular pattern is exhibited in a patient it is more likely that tongue is gradually affected and subsequently healed. While spiral patterns tend to be self-sustaining and will linger for a longer duration of time. These patterns result due to inhomogenities in the medium or due to external intervention in other excitable media. However, more studies are further needed [4]. The characteristic pattern of the lesions gives the appearance of a map on the dorsal surface of the tongue. Lateral margins and tip of the tongue are the most commonly involved sites followed by dorsal and ventral surfaces. If the lesions occur in different sites other than tongue, then the term ectopic geographic tongue is employed as first coined by Crooke in 1955. The extra glossal sites include the labial mucosa, buccal mucosa, gingival floor of the mouth, soft palate and uvula [4].

Microscopic examination reveals a thick keratin layer infiltrated by mixed inflammatory cell infiltrate, thin elongated rete ridges and epithelial oedema. The inflammatory cells produce small micro abscesses called Monro's abscesses in the keratin and spinous layer. Superficial areas may reveal candidal hyphae and spores [2] (Figure 3).

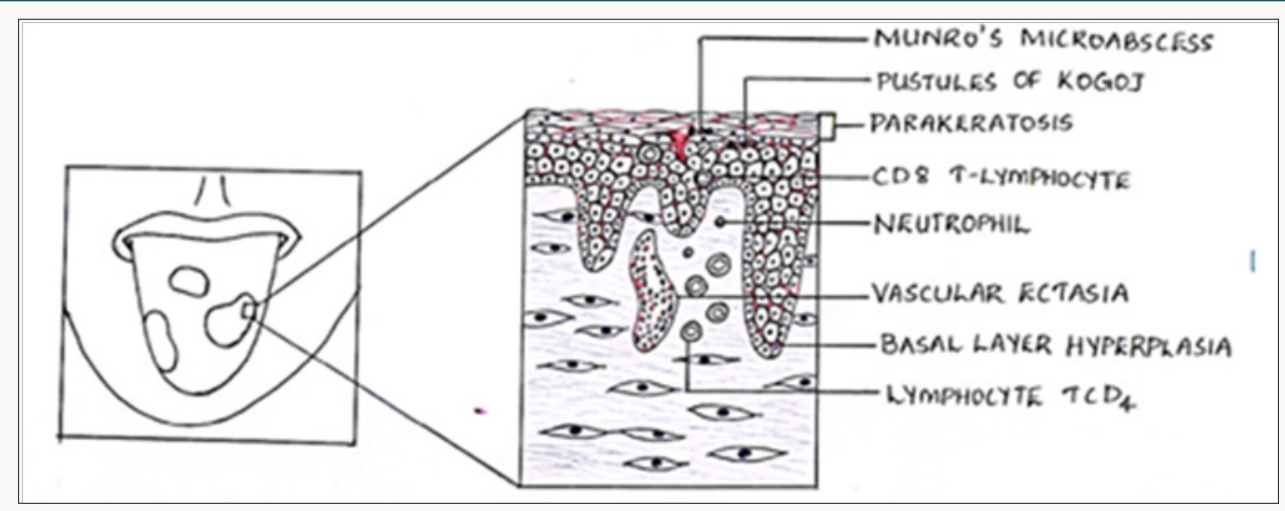

Figure 3: Histopathological features of Geographic tongue.

The diagnosis of GT is usually based on history and physical examination, except for atypical cases where histopathological examination is necessary. The lesions are characterized by migratory pattern, circinate aspect and mild pain [2].Management of GT depends upon the clinical presentation, the underlying etiology and should include reassuring the patients more so with cancer phobia about the benign nature of the disease [7]. A serious and persistent case of GT can be treated with systemic Cyclosporine and topical Tacrolimus[2]. 


\section{Conclusion}

Because it is a benign and generally asymptomatic lesion patients do not usually require treatment apart from reassurance [7]. Symptomatic treatment is based on the use of oral rinses containing anesthetics, topical corticosteroids, vitamin A, antihistamines and zinc supplements. Use of diphenhydramine, lidocaine rinse and sodium bicarbonate rinse has shown successful results in symptomatic cases of mild intensity [1]. It is recommended also to avoid contact with irritants and infectious factors, such as dentures and braces, which may exacerbate the situation. The patient should be instructed in relation to diet, avoiding acidic and spicy foods. In addition, patients should be instructed to always maintain good oral hygiene [2].

\section{References}

1. Mehta V (2017) Benign Migratory Glossitis: Report of a Rare Case with Review of Literature. J Dept of Pediatric and Preventive Dentistry 6(4): 00210 .
2. Picciani BLS, Domingos TA, Teixeira-Souza T (2016) Geographic tongue and psoriasis: clinical, histopathological, immunohistochemical and genetic correlation - a literature review. Anais Brasileiros de Dermatologia 91(4): 410-421.

3. Patil S, Kaswan S, Rahman F, Doni B (2013) Prevalence of tongue lesions in the Indian population. Journal of Clinical and Experimental Dentistry 5(3): e128-e132.

4. Nandini D B, Bhavana S B, Deepak B S, Ashwini R (2016) Paediatric Geographic Tongue: A Case Report, Review and Recent Updates. Journal of Clinical and Diagnostic Research : JCDR 10(2): ZE05-ZE09.

5. Gunashekar M (2014) Geographic tongue in monozygotic twins. J ClinDiagn Res 8(4): ZD01-ZD02.

6. Barton DH ,Spier SK, Crovello TJ (1982) Benign migratory glossitis and allergy. Pediatr Dent 4(3): 249-250.

7. Hooda A, Rathee M, Gulia J, Yadav S (2011) Benign migratory glossitis: a review. The Internet Journal of Family Practice 9(2): 1528-8358.
(C) This work is licensed under Creative To Submit Your Article Click Here: Submit Article

DOI: $10.32474 /$ IPDOAJ.2018.01.000106

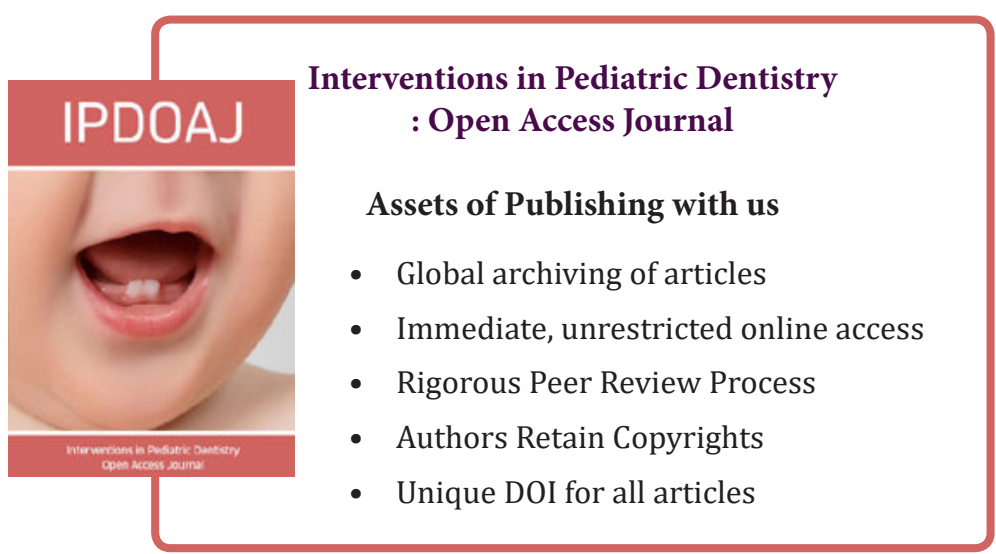

